\title{
Open-Access-Transformation in der Literaturwissenschaft
}

Open Access für exzellente Publikationen aus der Deutschen Literaturwissenschaft: Dank der Unterstützung von 39 wissenschaftlichen Bibliotheken können 2021 insgesamt neun literaturwissenschaftliche Neuerscheinungen transformiert und unmittelbar im Open Access veröffentlicht werden, ohne dass für Autorinnen und Autoren Publikationskosten entstehen.

Folgende Einrichtungen haben durch ihren Beitrag die Open-Access-Veröffentlichung dieses Titels ermöglicht:

Universitätsbibliothek Augsburg

Universitätsbibliothek Bayreuth

University of California, Berkeley Library

Staatsbibliothek zu Berlin - Preußischer Kulturbesitz

Universitätsbibliothek der Freien Universität Berlin

Universitätsbibliothek der Humboldt-Universität zu Berlin

Universitätsbibliothek Bielefeld

Universitäts- und Landesbibliothek Bonn

Universitätsbibliothek Braunschweig

Staats- und Universitätsbibliothek Bremen

Universitätsbibliothek der Technischen Universität Chemnitz

Universitäts- und Landesbibliothek Darmstadt

Sächsische Landesbibliothek - Staats- und Universitätsbibliothek Dresden

Universitätsbibliothek Duisburg-Essen

Universitäts- und Landesbibliothek Düsseldorf

Universitätsbibliothek Johann Christian Senckenberg, Frankfurt a. M.

Bibliothek der Pädagogischen Hochschule Freiburg

Niedersächsische Staats- und Universitätsbibliothek Göttingen

Universitätsbibliothek Greifswald

Universitätsbibliothek der FernUniversität in Hagen

Universitäts- und Landesbibliothek Sachsen-Anhalt, Halle (Saale)

Staats- und Universitätsbibliothek Hamburg Carl von Ossietzky

Gottfried Wilhelm Leibniz Bibliothek - Niedersächsische Landesbibliothek, Hannover

Universitäts- und Landesbibliothek Tirol, Innsbruck

Universitätsbibliothek Kassel - Landesbibliothek und Murhardsche Bibliothek der Stadt Kassel

Universitätsbibliothek der Universität Koblenz-Landau

Zentral- und Hochschulbibliothek Luzern

Universitätsbibliothek Marburg

Universitätsbibliothek der Ludwig-Maximilians-Universität München

Universitäts- und Landesbibliothek Münster

Bibliotheks- und Informationssystem der Carl von Ossietzky Universität Oldenburg

Landesbibliothek Oldenburg

Universitätsbibliothek Osnabrück

Universitätsbibliothek Trier

Universitätsbibliothek Vechta

Herzogin Anna Amalia Bibliothek, Weimar

Herzog August Bibliothek Wolfenbüttel

Universitätsbibliothek Wuppertal

Zentralbibliothek Zürich 